\title{
Pharmacokinetic Concentration Reason For Exclusion From Statistics
}

National Cancer Institute

\section{Source}

National Cancer Institute. Pharmacokinetic Concentration Reason For Exclusion From

Statistics. NCl Thesaurus. Code C119905.

A rationale for excluding a particular data point or subject from the pharmacokinetic concentration results statistical analysis. 